\title{
Editorial
}

\section{Clinical Factor 2012}

\author{
Richard Balon
}

Departments of Psychiatry and Behavioral Neurosciences and Anesthesiology, Wayne State University School of Medicine, Detroit, Mich., USA

As I have done twice in previous years [1, 2], I am presenting my subjective selection this time of 19 (primary) articles published in the previous year (i.e. in 2012) that I believe may provide some information to help the reader improve his/her clinical practice. This was originally proposed by Giovanni Fava [3] with the goal of selecting papers that I would subjectively perceive as having a high 'clinical factor' - the degree and extent to which a journal article provides information to the clinician that may improve his/her practice. As I noted before: 'Many may find them useful, some will find them less useful or useless. I am certainly biased, as is anybody else. I also cannot review all psychiatric journals - I focused only on most of the major ones. If there is no article selected from a certain major psychiatric journal, it does not necessarily mean that I have not reviewed it or skimmed through that journal. It may mean that I did not find, in my opinion, anything clinically very interesting' [1]. This year, I again expanded the number of journals I reviewed for clinically interesting and relevant articles. The journals that may or may not provide an article for this selection include the following: Acta Psychiatrica Scandinavica, American Journal of Psychiatry, Archives of General Psychiatry, British Journal of Psychiatry, Depression and Anxiety, European Psychiatry, General Hospital Psychiatry, Journal of Affective Disorders, Journal of Clinical Psychiatry, Journal of Clinical Psychopharmacology, Journal of Psychosomatic Research, Journal of Nervous and Mental Disease, Journal of Sex and Marital Therapy, Psychological Medicine, Psychosomatics and Psychotherapy and Psychosomatics. I view myself first and foremost as a clinician (though academically oriented) and I am, as most clinicians are, looking for a way to help patients, and thus looking for new, or innovative, or newly validated treatments. Thus that is part of my clinician's bias and that impacts my selection - the majority of the selected articles are on treatment-related issues or long-term outcome. I had originally selected more articles and it has been difficult to narrow them down to the final 19. I am sure I missed some interesting ones, but as noted, this is a subjective selection.

\section{First Episode of Psychosis: Detection and Prevention}

Detecting an illness in its earliest form and treating it and/or preventing the development of a full-blown disease through various interventions is a sound, reasonable, yet not always scientifically proven clinical strategy. In a study by Hegelstad et al. [4], 281 patients with the first episode of nonaffective psychosis were evaluated in $4 \mathrm{sec}$ tors in Norway and Denmark, using an early detection system (141 patients) and the usual detection system. One hundred and one of the early-detection patients and 73 of the usual-detection patients were followed up to 10 years (last follow-up at 10 years). A significantly higher number

\section{KARGER}

E-Mail karger@karger.com www.karger.com/pps
C 2013 S. Karger AG, Basel

0033-3190/13/0824-0204\$38.00/0
Richard Balon, MD

Departments of Psychiatry and Behavioral Neurosciences and Anesthesiology Tolan Park Building, Third Floor

3901 Chrysler Service Dr., Detroit, MI 48201 (USA)

E-Mail rbalon@wayne.edu 
of early-detection patients (30.7\%) compared to the usual-detection patients (15.1\%) fulfilled recovery criteria. Although the mechanism of this is unknown and speculative, early detection of the first episode of psychosis appears to increase the chances of milder deficits and superior functioning. As the authors noted, early detection provides a lower threshold for entering treatment irrespective of the duration of untreated psychosis (i.e. patients do no need to display suicidality or dramatic symptoms to be 'allowed' into the treatment system). In addition, as the authors noted, the recovery rates of early-detection patients were higher largely because of higher employment rates of these patients. This may suggest that the role of involvement in the work process and vocational rehabilitation in recovery may be larger than previously thought (word of caution: the direction of 'causality' has not been explored here and the sample was small). (This study was supported by Health West, Norway, the Norwegian National Research Council, the Norwegian Department of Health and Social Affairs, The National Council for Mental Health/Health and Rehabilitation, Rogaland and Oslo Counties; the Regional Health Research Foundation for Eastern Region, Denmark, and numerous other agencies, including Lundbeck Pharma, Eli Lilly, Janssen-Cilag, NARSAD, NIMH to individual researchers.)

In a multicenter, prospective, randomized trial, Bechdolf et al. [5] assigned 128 patients in early initial prodromal state (patients presenting with at least 1 of 10 thought or perceptional symptoms) to either integrated psychological intervention or supportive counseling. Integrated psychological intervention consisted of individual cognitive-behavioral therapy (CBT), modified social skills training, cognitive remediation and multifamily psychoeducation. Both interventions were delivered over a 12-month period and follow-up was conducted for up to 24 months after intake. The integrated psychological intervention was superior to psychological counseling in preventing progression into subthreshold at 12-month follow-up (3.2 vs. $16.9 \%, \mathrm{p}=0.008$ ) and at 24 -month follow-up ( 6.3 vs. $20.0 \%, p=0.019$ ). The time to conversion for the entire study period was significantly shorter for the supportive counseling group than for the integrated psychological counseling group. (This study was funded by the German Federal Ministry for Education and Research grant to the German Research Network on Schizophrenia.)

As part of a 2-year prospective, randomized, open-label, controlled trial of the effects of maintenance antipsychotic treatment versus guided discontinuation in 124 patients with first-episode nonaffective psychosis, Faber et al. [6] studied the association of cannabis use and measures of psychopathology and social role functioning. Interestingly, in contrast with some other studies, continued cannabis use was not associated with symptomatic or functional remission and clinical recovery. However, cannabis use was related to certain aspects of role functioning (economic and social activities), but not to psychopathology. The authors felt that though the role of cannabis in the outcome of psychosis is probably modest, the continuous use of cannabis after the onset of firstepisode psychosis should be discouraged. (This study was funded by a grant from the Netherlands Organization for Health Research and Development, the Foundation for the Support of the Society of Christian Care of the Nervous and Mentally Ill, the Foundation 'De Open Ankh', and by unrestricted grant from Eli Lilly.)

The results of these studies seem to confirm an emerging strategy for the management of the first-episode psychosis - combination of early detection, intensive psychological intervention (and possible targeted medication) and refraining from cannabis and other substances of abuse. The warning about refraining from cannabis abuse may sound superfluous, but it remains very important during the era of multiple movements for legalizing the use of cannabis and use of medical cannabis in the management of pain.

\section{Treatment of Schizophrenia}

Treatment of schizophrenia is a long-term affair including frequent use of antipsychotic medication and low use of psychotherapies, and associated with complications and frequent lack of substantial positive functional and other outcomes. Grant et al. [7] examined whether cognitive therapy (CT) may address, at least in part, the issue of limited improvement in functional outcomes in low-functioning patients with schizophrenia. They studied 60 low-functioning, neurocognitively impaired patients with schizophrenia in a single-center, 18-month, randomized, single-blind, parallel-group trial of CT (usually 50-min weekly sessions) plus standard treatment versus standard treatment (at minimum, this consisted of antipsychotic medication). They found that patients assigned to CBT plus standard treatment had better functioning, reduced avolition-apathy, and improved positive symptoms (hallucinations, delusions, disorganization) relative to patients who received standard treatment only. Though the study had some limitations (use of the Glob- 
al Assessment Scale as the primary outcome measure; more frequent contacts with the CBT patients might raise the possibility of nonspecific contacts contributed to the positive effects), these findings suggest that there may be other venues to help chronic schizophrenia patients improve their quality of life, and negative symptomatology so difficult to treat. (This study was supported by a Distinguished Investigator Award from the NARSAD and by grants from the Heinz Foundation and the Barbara and Henry Jordan Foundation.)

The prevailing standard of care is that schizophrenia patients need to be treated with antipsychotic medication continuously throughout their life. Harrow et al. [8] examined this assumed paradigm in a 20-year longitudinal study of 70 patients with schizophrenia and 69 patients with mood disorders. These patients were prospectively assessed at baseline and then 6 times over the next 20 years for major symptoms, psychosocial functioning, personality, attitudinal variables, neurocognition and treatment. There was no single uniform treatment plan that applied to all patients. The authors found that not all schizophrenia patients in their study needed treatment with antipsychotics continuously throughout their lives. Schizophrenia patients who were not on antipsychotics for prolonged periods were a self-selected group with better internal resources associated with greater resiliency, who had also better prognostic factors at baseline, better premorbid developmental achievements, less vulnerability to anxiety, better neurocognitive skills, less vulnerability to psychosis and experienced more periods of recovery. Interestingly, the data from this study did not provide any evidence of whether a very long-term use of antipsychotic medication produces undesirable effects for some schizophrenia patients. (This study was supported, in part, by United States Public Health Service grants and grant from the NIMH.)

Using antipsychotics during pregnancy is usually considered relatively safe, especially when using older antipsychotics. As our knowledge of the effects of exposure to the newer antipsychotics during pregnancy is limited, Boden et al. [9] investigated the effects of maternal use of antipsychotics during pregnancy on gestational diabetes and fetal growth in a population-based cohort study comparing women exposed and not exposed to antipsychotics during pregnancy. They used the Swedish national health registers for all women giving birth in Sweden between July 1, 2005 and December 31, 2009, with exposure to antipsychotics defined as prescriptions filled. They obtained data on 169 women exposed to olanzapine and/or clozapine (considered to be the obesogenic and diabetogenic an- tipsychotics), 338 women on other antipsychotics (quetiapine, risperidone, flupentixol, haloperidol, aripiprazole, perphenazine, zuclopenthixol, ziprasidone, chlorprothixene, fluphenazine, pimozide lumped together), and 357,696 women on no antipsychotics. Gestational diabetes was more than twice as common in mothers who used antipsychotics than in the total population of pregnant women. The unadjusted odds ratios were of similar magnitude in both the olanzapine/clozapine group and other antipsychotics group. The odds ratios remained similar after adjusting for birth order, maternal age, country of birth, cohabitation, smoking and height. Infants exposed to antipsychotics had a more-than-doubled risk of being small for gestational age, regardless of the drug group. However, the increased risk of giving birth to a small-for-gestational-age infant observed in women treated with antipsychotics during pregnancy is probably due to confounding factors, such as smoking. The study was a bit limited by the relatively small numbers of women in both antipsychotics groups and by including risperidone and quetiapine in the second group (though secondary analyses did not find any impact of this grouping). Nevertheless, it seems obvious that women on antipsychotics during pregnancy should be monitored for gestational diabetes carefully. (This study was supported by unrestricted grants from Lenander's Foundation and Gillbergska Foundation.)

Findings from these studies support individualized treatment of schizophrenia, with targeted intermittent use of medication dependent on symptomatology, increased use of psychological therapies such as CBT and a careful use of antipsychotics during pregnancy.

\section{Management of Posttraumatic Stress Disorder}

Posttraumatic stress disorder (PTSD), with its welldefined onset (trauma), is a good candidate for secondary prevention - intervention to prevent development of PTSD symptomatology after trauma. Studying the efficacy of such interventions has been difficult, as not everybody develops PTSD symptoms following trauma. Studying secondary prevention is also difficult from an ethical standpoint, as in a 'gold standard' design - a doubleblind, placebo-controlled study - some patients do not receive intervention which may possibly prevent the development of a disorder. Nevertheless, preventing PTSD is a pressing need and some pharmacological prevention studies have not been successful. Thus, Shalev et al. [10], in a complex and complicated equipoise-stratified ran- 
domized controlled study, compared early and delayed prolonged exposure (PE and delayed PE), CT and escitalopram (20 mg/day) in possible prevention of PTSD. The groups included 63 patients who received 12 weekly sessions of $\mathrm{PE}, 40$ patients who received CT, 23 patients on escitalopram, 23 patients on placebo and 93 patients on waiting list for 12 weeks. Treatment started on average 30 days after the traumatic event. Waiting list participants with PTSD (41 of 57 who still had PTSD symptomatology after 3 months on the waiting list) received PE about 151 days after the traumatic event (delayed PE). Subjects were evaluated for PTSD symptomatology at 5 and 9 months. At 5 months, the prevalence of PTSD in the PE (21.4\%) and CT (18.2\%) groups was significantly lower than in the waiting list (58.2\%), escitalopram (61.9\%) and placebo (55.6\%) groups. At 9 months, the prevalence of PTSD in the PE (21.2\%), CT (22.8\%) and waiting list (22.9\%) groups was lower than in the escitalopram (42.1\%) and placebo (47.1\%) groups. These results show that psychological interventions such as PE (even delayed one) and CT have some preventive effect on PTSD symptomatology. The study had some limitations, such as small treatment groups, high pre-enrollment attrition rates, and the use of a sample of civilian survivors of a single, short traumatic event. It also seems that the effect of psychological interventions may have plateaued after 5 months, while the effect of escitalopram and placebo (!) was delayed. (The study was supported by Jerry Lee Foundation in Philadelphia, Pa., a grant from the Jewish Federation of New York, a grant from NIMH and an investigator-initiated grant from Lundbeck Pharmaceuticals Ltd.)

PTSD, like many other disorders, may also impact intimate relationships and intimate relationship factors may impact individual PTSD treatment outcomes. Monson et al. [11] studied the efficacy of cognitive-behavioral conjoint therapy for PTSD (a manualized couple therapy delivered to patients with PTSD and their significant others to simultaneously treat PTSD symptoms and enhance relationship satisfactions) (20 couples, 15 sessions) comparing it to the waiting list condition in a randomized fashion. The study was conducted in Boston, Mass., USA and Toronto, Ont., Canada. PTSD symptom severity and intimate relationship satisfaction both improved significantly more in the cognitive-behavioral conjoint therapy group than in the waiting list group and treatment effects were maintained at 3-month follow-up. The interpretation of the results is limited by the small sample size (not all couples finished the study), sample heterogeneity, careful selection of the sample (mostly white, employed, almost no substance abuse at baseline), no assessment of axis II comorbidity and several other factors. It is surprising that this study was published in spite of its numerous limitations. The role of cognitive-behavioral conjoint therapy for PTSD definitely needs further evaluation as this study provides more questions than answers. (This study was supported by an NIMH grant.)

Substance abuse and PTSD frequently co-occur and co-occurring substance abuse frequently makes the treatment of PTSD (and other conditions) more difficult and complicated. Mills et al. [12] in Sydney, Australia, attempted to determine whether an integrated treatment for PTSD and substance dependence, concurrent treatment of PTSD and substance use disorders using prolonged exposure (COPE), can achieve greater reduction in PTSD and substance dependence symptom severity than the usual treatment for substance dependence. Participants were randomly assigned to COPE $(\mathrm{n}=55 ; 13$ individual 90-min sessions) plus usual treatment, or usual treatment alone ( $\mathrm{n}=48$; any type of substance abuse treatment, including outpatient counseling, inpatient or outpatient detoxification, residential rehabilitation, and pharmacotherapies such as methadone, buprenorphine, naltrexone etc.). There was a significant reduction in PTSD symptom severity for both treatment groups from baseline to 9-month follow-up; however, COPE plus usual treatment had a significantly greater reduction of PTSD symptom severity. There were no significant differences in improvement of severity of substance dependence, or changes in substance use, depression, or anxiety. However, as Najavits [13] points out in an accompanying editorial, both study conditions still had notable impairment in all domains assessed, despite the improvements from baseline. (The study by Mills et al. was funded by the Australian National Health and Medical Research Council grant.)

New treatment/prevention modalities for PTSD (and comorbid substance abuse) are being tested. However, as Najavits [13] points out:

Comparative studies of PTSD therapies find that they rarely outperform each other. Thus, the cost and appeal of treatment to clinicians and patients, their intensity of intervention, and clinical setting and training issues may ultimately be as or more relevant than comparative efficacy in choosing a course of treatment for PTSD.

She [13] also comments extensively on what was left out from the report of Monson et al. [11] - no data on how concurrent therapies may have affected outcomes, whether the couples were compensated financially for their participation in this trial and that neither the patients assigned to the waiting list condition nor patients who dropped out of treatment were included in the fol- 
low-up assessment. Additionally, the generalizability of this study remains unclear [13].

Finally, Najavits [13] also points out that it is not clear how long the treatment needs to be maintained to produce enduring positive outcomes. New treatments are clearly available, but their significance, applicability and usefulness remain unclear.

\section{Treatment of Obsessive-Compulsive Disorder}

CBT is considered to be the first-line evidence-based treatment of obsessive-compulsive disorder (OCD) with response rates of $50-70 \%$. However, as Andersson et al. [14] and many others point out, access to CBT is limited, mainly due to lack of well-trained CBT therapists and their geographic maldistribution. One option to increase the availability of CBT is Internet-based CBT (ICBT) with therapist support. Andersson et al. [14] tested the efficacy of ICBT for OCD in a randomized controlled trial of 101 OCD patients. They were randomly assigned to either 10 weeks of ICBT (50 subjects) or to online nondirective supportive therapy (51 subjects). The ICBT therapists had no face-to-face contact with patients and communicated with them online after being contacted via short mobile text messages. The supportive therapy consisted of access to an e-mail function integrated in the treatment platform, through which participants could communicate with a therapist. There were no active treatment components, such as self-help texts or worksheets. Both treatments led to significant improvement in OCD symptomatology [measured by the Yale-Brown Obsessive Compulsive Scale (Y-BOCS)]; however, ICBT resulted in larger improvement and the proportion of participants showing clinically significant improvement was higher in the ICBT group (60\%) than in the supportive therapy group (6\%). The results in the ICBT group were sustained at 4-month follow-up. The limitations of the study include higher phone contact numbers with the control group (possible nonspecific factors effect), and the fact that the controls were crossed over to ICBT after 10 weeks and thus follow-up observation comparisons were not possible. Finally, patients were largely self-referred and it is not clear whether they represented the typical OCD population or a more computer-apt subpopulation. (This study was supported by the Stockholm County Council, the Karolinska Institutet, the Swedish Research Council and the Swedish Society of Medicine.)

As OCD is frequently difficult to treat or treatmentresistant, the question is what to do when the first-step treatment (mostly CBT) does not work. Van Balkom et al. [15] studied 48 OCD patients who were nonresponsive to exposure in vivo with response prevention (ERP) (out of 118 patients who received 12 weeks of ERP). These nonresponders were randomly assigned to 12 weeks of either CT (22 patients, weekly sessions for 45 $\mathrm{min}$ ) or fluvoxamine (26 patients, up to $300 \mathrm{mg} /$ day). Actually, the data of only 45 patients were analyzed, as $50 \%$ of patients refused fluvoxamine and $13 \%$ of patients refused CT after randomization. In this remaining sample, fluvoxamine was significantly superior to CT (in the 17 CT group subjects, the Y-BOCS scores remained virtually unchanged, while in the 13 fluvoxamine subjects the scores decreased from $26.2 \pm 6.6$ to $19.2 \pm 8.0$ ). The interpretation of the results is certainly limited by the small sample size, the selective dropout rate in the fluvoxamine group and the lack of placebo control. However, the results suggest that after the failure of ERP, it may be more prudent to try medications rather than CT. (Funding source of this study is unclear, but the authors report no financial or personal relationship or affiliation that could inappropriately influence their decisions, work or this article.)

Finally, Albert et al. [16] in Turin and Rome studied the effectiveness of routinely delivered CBT as an adjunct to medication in real-world patients with incomplete response to medication. They enrolled 118 patients with partial but incomplete response (defined as Y-BOCS reduction $<25 \%$ ) to at least 12 weeks of $\geq 40 \mathrm{mg}$ /day of citalopram, fluoxetine and paroxetine, $\geq 100 \mathrm{mg} /$ day of clomipramine, $\geq 20 \mathrm{mg} /$ day of escitalopram, $\geq 200 \mathrm{mg} /$ day of fluvoxamine and sertraline, and $\geq 250 \mathrm{mg} /$ day of venlafaxine. Patients received the same medication and dosage during the trial and the CBT duration was not fixed in advance (the mean duration was 7.13 months). The mean reduction in Y-BOCS scores was $17.9 \%$ in 3 months and $29.3 \%$ in 6 months, the responder rates were $32.8 \%$ at 3 months and $58 \%$ at 6 months and the remitter rates were $15.1 \%$ at 3 months and $31.1 \%$ at 6 months. Though there was no control group, no randomization and the patients were self-selected, it seems that the addition of CBT for partial OCD responders to medication in routine clinical practice may work. (The funding of this study was not specified.)

These 3 studies expand our knowledge on the practicality of treatment of OCD, suggesting that ICBT may be useful in this indication, that the combination of medication and CBT may be useful in clinical practice and that CT-resistant OCD may respond to fluvoxamine. 


\section{Depression: Added Risks in Comorbidity, and Treatment Issues}

Depression is known to increase the risk of some physical illnesses or at least worsen their course and outcome. The relationship between depression and diabetes mellitus, as Katon et al. [17] point out, is bidirectional - 'with depressive episodes developing earlier in life leading to an increased risk of diabetes and adult-onset diabetes increasing the subsequent risk of depression'. As some studies found that depression and diabetes independently increase the risk of dementia, Katon et al. [17] decided to examine whether patients suffering from both depression and diabetes have even more increased risk of dementia. They examined a sample of 19,239 diabetes registry members 30-75 years of age from a large, integrated, nonprofit managed care setting in Northern California (Kaiser Permanente). The cases of depression were identified using the Patient Health Questionnaire score of at least 10, ICD-9 diagnoses of depression and/or antidepressant prescription in the 12 months prior to baseline. Dementia diagnoses were only based on ICD-9 diagnoses during years 3-5 after baseline. During the 3- to 5-year post-baseline period, $2.1 \%$ patients with comorbid depression and diabetes (incidence rate of 5.5 per 1,000 person-years) and $1.0 \%$ of patients with diabetes alone (incidence rate of 2.6 per 1,000 person-years) developed dementia. Thus, the risk of dementia doubled in patients in comorbid depression and diabetes when compared with patients with diabetes alone. The numbers were small, though, and the study was limited by lack of controls without diabetes and the study being done in one large 'setting' in one geographic area. (This study was supported by an NIMH grant and by National Institute of Diabetes and Digestive and Kidney grants. Dr. Katon has received honoraria from various pharmaceutical companies; however, this was not related to this study.)

Older patients with coronary artery disease are at greater risk for developing depression and also at greater risk for developing long-term cognitive decline. It is not known, however, whether depressive symptoms exacerbate the risk for cognitive decline in these patients. Freiheit et al. [18] from Calgary, Alta., Canada, examined the association between the stability of depressive symptoms and cognitive decline for 30 months in 350 patients (73.3\% males) undergoing nonemergent catheterization/ coronary angiography without previous revascularization. Depressive symptomatology was evaluated at baseline, and at 6 and 12 months using the 15-item Geriatric Depression Scale. Neurocognitive outcome measures in- cluded the Brief Visuospatial Memory Test-Revised, Consortium to Establish a Registry for Alzheimer's Disease Test of Verbal Learning and Memory, Controlled Oral Word Association and Animal Naming Test, Trail Making Test and Mini-Mental State Examination. The neurocognitive tests were administered at baseline, 6,12 and 30 months. In order to explore whether any changes were modified by the presence of the apolipoprotein $\mathrm{E} \varepsilon$ allele, the apolipoprotein E genotype was identified. Patients with persistent depressive symptoms showed significantly greater decline at 30 months in attention/executive function, verbal fluency and global cognition compared with those of no or baseline-only depressive symptoms. Interestingly, for global cognition, a significantly greater decline was evident for those with persistent depressive symptoms and the apolipoprotein E $\varepsilon 4$ allele. Though the generalizability of these findings is limited, it seems that the presence of persistent depressive symptoms increases the risk of cognitive decline in patients with coronary artery disease. [This study was supported by the Canadian Institutes of Health Research Institute of Aging, the Medical Services (Alberta) Incorporated Foundation, and the Brenda Strafford Foundation Chair in Geriatric Medicine. The funding organizations played no role in this study besides funding it.]

The possible association of suicidal thoughts and behavior with antidepressant treatment has been a controversial issue over the past 2 or more decades since the original report by Teicher et al. [19] in 1990. Subsequently, the US FDA issued a black box warning for antidepressants and suicidal thoughts and behavior in children and young adults. Gibbons et al. [20] recently reanalyzed all intent-to-treat person-level longitudinal data with regard to suicidal thoughts and behavior in youth, adult, and geriatric populations of major depressive disorder from 12 adult, 4 geriatric, and 4 youth randomized controlled trials of fluoxetine and 21 adult trials of venlafaxine with a focus on the mediating effect of changes in depressive symptoms. The data obtained from sponsored trials included suicide item rating from the Children Depression Rating Scale-Revised and the Hamilton Depression Rating Scale, as well as adverse reports of suicide attempts and suicide during active treatment in 9,185 patients (fluoxetine: 2,635 adults, 960 geriatric patients, 708 youth; venlafaxine: 2,421 adults with immediate-release venlafaxine and 2,461 adults with extendedrelease venlafaxine). Fluoxetine and venlafaxine decreased suicidal thoughts and behavior in adult and geriatric patients when compared to placebo. No difference was found in the youths on fluoxetine, though de- 
pressive symptomatology improved. In adults, the reduction in suicide ideation and attempts was mediated through a reduction in depressive symptoms. No evidence of increased suicide risk was observed in youths receiving active medication. As the authors pointed out, their findings emphasize the need to successfully treat major depressive disorder to lower the suicide risk, in adult and geriatric population. This study did not replicate the FDA's findings of increased suicide risk with fluoxetine treatment in youths. The study was limited by relying on a single ordinal clinician rating of suicidal behavior, supplemented by adverse reports of suicide attempts or suicides. However, most studies in this area used similar data. The other limitation is the usual exclusion of suicidal patients from randomized controlled trials used here - but suicidal thoughts and behavior rates were highest at baseline and 9/20 suicide attempts occurred prior to treatment initiation. (This study was supported by NIMH and Agency for Healthcare Research and Quality grants. Dr. Gibbons served as an expert witness for the US Department of Justice, Wyeth and Pfizer Pharmaceuticals in cases related to antidepressants and anticonvulsants and suicide. Dr. Brown's program received funding from JDS Pharmaceuticals. Dr. Mann received grant support from GSK and Novartis.)

As numerous studies found that treatment of depression with antidepressants is frequently not very successful, various augmentation strategies have been advocated and promoted. One of them has been the adjunctive treatment of depression with aripiprazole. In a study with an interesting and complex design, M. Fava et al. [21] assessed the efficacy of low-dose aripiprazole added to various antidepressant treatments (citalopram, escitalopram, desvenlafaxine, fluoxetine, paroxetine, sertraline, venlafaxine, and others) of major depression in patient with inadequate response to prior antidepressant treatment. Their study of 255 patients ( 221 in primary efficacy sample) had an interesting design - patients were assigned randomly to adjunctive treatment with aripiprazole $2 \mathrm{mg}$ /day or placebo across two 30-day phases with a 2:3:3 randomization ratio to adjunctive treatment as follows: drug/drug (aripiprazole $2 \mathrm{mg} /$ day for 30 days and then $5 \mathrm{mg} /$ day for 30 days); placebo/drug (placebo for 30 days and aripiprazole $2 \mathrm{mg}$ /day for 30 days) and placebo/placebo (placebo for 30 days and again for 30 days). The difference between aripiprazole and placebo at any endpoint was small, not statistically significant and definitely not clinically significant. The differences in side effects were minimal, though aripiprazole was associated with more constipation, weight gain and dry mouth, but surprisingly not with more akathisia. Though the authors concluded that 'This study provides clear support for the tolerability of low-dose aripiprazole as an added to antidepressant therapy-augmenting agent, with marginal efficacy', this is basically a negative study. The authors note the main limitation of their study: all their patients had historical failures to antidepressant therapy, whereas the patients in 3 previous positive studies of aripiprazole augmentation with inadequate response to antidepressant therapy all had at least 1 prospective failure. This may be a limitation, but it is also a reflection of clinical reality. (This investigator-initiated study was supported by Bristol-Myers Squibb, which also provided blinded study medication.)

While this was a basically negative study, it is important for any clinician and anyone conducting clinical trials because of the study design. In an accompanying editorial, Tomba [22] points out that this study is the first one to use the sequential parallel comparison design, which used 2 phases of treatment. In the first phase, patients were assigned to placebo or active treatment (more to placebo in this study). In the second phase, the active treatment (in this study aripiprazole) dose was increased in those who received it during the first phase, whereas those who were on placebo in the first phase received a low dose of active treatment (here $2 \mathrm{mg}$ of aripiprazole). There was still a placebo/placebo group. As Tomba [22] states, there are two advantages of this design:

One is that active treatment/placebo randomization in the second phase excludes not only the patients responding to the early effects of placebo, as would happen with the customary brief placebo washout periods, but also the patients who respond to unspecified ingredients (such as intensity of the patient clinician interaction), which may occur only at some later point in time.

The other advantage is that this design allows not only comparing patients between different groups, but also patients within groups. It allows maximizing the detection of differences between active treatment and placebo (not detected here). (No support or conflict of interest noted for this editorial, though Dr. Tomba co-authored several articles on the sequential parallel comparison design.)

It seems that depression should be treated vigorously in patients with comorbid diabetes or with comorbid coronary artery disease, as the treatment may possibly decrease the increased risk of cognitive impairment in these patients and the vigorous treatment may not increase the risk of suicidal thoughts and/or behavior in these patients. However, it seems that aripiprazole may not be the way to augment antidepressant treatment of depression in patients with previous treatment failures. 


\section{Depression and the Heart - Unresolved Issues?}

Depression after myocardial infarction is common and is associated with poor cardiovascular prognosis. There is actually some evidence that depressed myocardial infarction patients are at particular risk of poor prognosis when the depressive episode has an onset after rather than before the acute cardiac event [23]. Zuidersma et al. [23] studied 442 depressed and 325 nondepressed myocardial infarction patients to find out whether an increase in the number of depressive symptoms after myocardial infarction is associated with new cardiac events. They used the Composite International Diagnostic Interview, which, for the goal of this study, was extended with additional questions to assess the presence of each ICD-10 symptom of depression during the 4 weeks before and after myocardial infarction. New fatal and nonfatal cardiac events occurring after the depression interview were included as endpoints and were assessed by patient interview, hospital records and data from treating specialists (follow-up period up to 2.5 years after myocardial infarction). Each additional increase of one depression symptom was associated with a $15 \%$ increased risk of new cardiac event. Interestingly, this finding was stronger for nondepressed than for depressed patients. There was no association with the number of depressive symptoms before myocardial infarction. The increase of depressive symptoms after myocardial infarction may be developed as a prognostic factor. (This study was supported by a VIDI grant from the Dutch Medical Research Council, the MIND-IT is sponsored by the Netherlands Heart Foundation and received educational grants from Organon and Lundbeck. DepreMI is sponsored by a grant from the Netherlands Organization for Scientific Research.)

Similarly, Myers et al. [24] evaluated a cohort of 632 patients 65 years old or older for the presence of depressive symptoms at the time of their admission for the firstever myocardial infarction. Depressive symptoms were evaluated using the Beck Depression Inventory. The cohort was followed up for 10-13 years. Postmyocardial infarction hospital admissions and secondary prevention measures (attendance at cardiac rehabilitation; smoking status and its change; adherence to medications; leisure time physical activity) and their relationship to depressive symptoms were examined to assess the burden of postmyocardial infarction depression on patients and the health care system. Depressive symptoms were significantly associated with days of hospitalization during the follow-up and the association was stronger for cardiacrelated admissions than for noncardiac admissions. De- pressed patients were less likely to stop smoking, to be physically active and to participate in cardiac rehabilitation. Though the study had several limitations (use of a self-report rating scale rather than clinical interview for depression; single-point depressive symptoms evaluation; lack of data about mental health history and previous episodes of depression), the results nevertheless again point out the negative impact of depression on myocardial infarction outcome/consequences. (This study was supported in part by the Israel National Institute for Health Policy and Health Service Research.)

The negative impact of depression on the outcome of acute coronary events seems to be clear and thus patients should be monitored for the development of depression and subsequently vigorously treated when depression present. However, as Thombs and Ziegelstein [25] warn us, one should not conclude that the presence of coronary artery syndrome without depression requires preventive administration of antidepressants. To the argument that the potential risk and considerable cost of preventive treatment are reasonable when one considers the risk of developing depression in acute coronary syndrome patients, Thombs and Ziegelstein [25] say that this 'presupposes either that a case of depression would not be recognized once it develops or that by the time it is recognized, it would be too late for treatment to be beneficial or to prevent the adverse consequences of the disease. There is no evidence to support either assumption'. The debate on the preventive use of antidepressants in 'prevention' of depression in coronary artery syndrome patients is changing in a similar way as the debate on using antidepressants in patients treated with interferon. (Dr. Thombs is supported by the Canadian Institutes of Health Research and an the Fonds de la Recherche en Sante Quebec and Dr Ziegelstein is supported by the National Center for Complementary \& Alternative Medicine grant and the Miller Family Scholar Program at Johns Hopkins Center at Innovative Medicine.)

\section{Attention-Deficit/Hyperactivity Disorder}

Poor outcomes in various areas of functioning have always been one of the arguments for the treatment of attention-deficit hyperactivity disorder (ADHD) and also supported the validity of the ADHD diagnosis. Klein et al. [26] in their prospective, 33-year follow-up study of 135 white men with ADHD in childhood (diagnosis at the age of 8), free of conduct disorder, and 136 men without childhood disorder found that probands (with ADHD) had significantly worse educational, occupational, eco- 
nomic, and social outcomes; high rates of divorce, and higher rates of ongoing ADHD, but no more mood or anxiety disorders than comparison participants. Ongoing ADHD was weakly related to ongoing substance use disorders and antisocial personality disorder. Unfortunately, the generalization of the results is limited by the fact that the subjects were all white males of average intelligence and had the diagnosis of the combined-type of ADHD. Though this unique study does not present any data on the impact of possible treatment on its probands either, the results underscore the importance of extended monitoring and treatment of children with ADHD. Also, the course of childhood ADHD revealed a consistent clinical pattern from late adolescence into adulthood, the longitudinal course supported the diagnostic validity of ADHD as defined in this sample. (This research was supported by grants from the NIMH and by a grant from the National Institute on Drug Abuse.)

\section{Disclosure Statement}

The author declares no conflict of interest.

\section{References}

1 Balon R: Clinical factor 2010. Psychother Psychosom 2011;80:195-198.

-2 Balon R: Clinical factor 2011. Psychother Psychosom 2012;81:199-205.

3 Fava GA: The clinical factor. Psychother Psychosom 2011;80:1-3.

$\checkmark 4$ Hegelstad WT, Larsen TK, Auestad B, Evensen J, Haahr U, Joa I, Johanessen JO, Langeveld J, Melle I, Opjordsmoen S, Rossberg JI, Rund BR, Simonsen E, Sundet K, Vaglum $\mathrm{P}$, Friis S, McGlashan T: Lung-term follow-up from the TIPS early detection in psychosis study: effect on 10-year outcome. Am J Psychiatry 2012;169:374-380.

5 Bechdolf A, Wagner M, Ruhrmann S, Harrigan S, Putzfeld V, Pukrop R, BrockhausDumke A, Berning J, Janssen B, Decker P, Bottlender R, Maurer K, Moller H-J, Gaebel W, Hafner H, Maier W, Klosterkotter J: Preventing progression to first-episode psychosis in early initial prodromal states. Br J Psychiatry 2012;200:22-29.

-6 Faber G, Smid HGOM, Van Gool A, Wunderink L, van den Bosch R, Wiersma D: Continued cannabis use and outcome in first-episode psychosis: data from a randomized, open-label, controlled trial. J Clin Psychiatry 2012;73:632-638.

7 Grant PM, Huh GA, Perivoliotis D, Stolar NM, Beck AT: Randomized trial to evaluate the efficacy of cognitive therapy for low-functioning patients with schizophrenia. Arch Gen Psychiatry 2012;69:121-127.

8 Harrow M, Jobe TH, Faull RN: Do all schizophrenia patients need antipsychotic treatment continuously throughout their lifetime? A 20-year longitudinal study. Psychol Med 2012;42:2145-2155.

9 Boden R, Lundgren M, Brandt L, Reutfors J, Kieler H: Antipsychotics during pregnancy. Relation to fetal and maternal metabolic effects. Arch Gen Psychiatry 2012;69:715-721.

-10 Shalev AY, Ankri Y, Israeli-Shalev Y, Peleg T, Adessky R, Freedman S: Prevention of posttraumatic stress disorder by early treatment. Results from the Jerusalem Trauma Outreach and Prevention Study. Arch Gen Psychiatry 2012;69:166-176

11 Monson CM, Fredman SJ, Macdonald A, Pukay-Martin ND, Resic PA, Schnurr PP: Effects of cognitive-behavioral couple therapy for PTSD. A randomized controlled trial. JAMA 2012;308:700-709.

12 Mills KL, Teesson M, Back SE, Brady KT, Baker AL, Hopwood S, Sannibale C, Barrett EL, Merz S, Rosenfeld J, Ewer PL: Integrated exposure-based therapy for co-occurring posttraumatic stress disorder and substance dependence. A randomized controlled trial JAMA 2012;308:690-699.

13 Najavits LM: Expanding the boundaries of PTSD treatment. JAMA 2012;308:714-716.

14 Andersson E, Enander J, Andren P, Hedman E, Ljotsson B, Hursti T, Bergstrom J, Kaldo V, Lindefors N, Andersson G, Ruck C: Internetbased cognitive behavior therapy for obsessive-compulsive disorder: a randomized controlled trial. Psychol Med 2012;42:2193-2203.

15 Van Balkom AJLM, Emmelkamp PMG, Eikelenboom M, Hoogendoorn AW, Smit JH, van Oppen P: Cognitive therapy versus fluvoxamine as a second-step treatment in obsessive-compulsive disorder nonresponsive to first-step behavior therapy. Psychother Psychosom 2012;81:366-374.

16 Albert U, Aguglia A, Bogetto F, Cieri L, Daniele M, Maina G, Necci R, Parena A, Salvati L, Tundo A: Effectiveness of cognitive-behavioral therapy addition to pharmacotherapy in resistant obsessive-compulsive disorder; a multicenter study. Psychother Psychosom 2012;81:383-385.

17 Katon W, Lyles CR, Parker MM, Karter AJ, Huang ES, Whitmer RA: Association of depression with increased risk of dementia in patients with type 2 diabetes. The Diabetes and Aging Study. Arch Gen Psychiatry 2012; 69:410-417.

18 Freiheit EA, Hogan DB, Eliasziw M, Patten SB, Demchuk AM, Faris P, Anderson T, Galbraith D, Parboosingh JS, Ghali WA, Knudtson M, Maxwell CJ: A dynamic view of de- pressive symptoms and neurocognitive change among patients with coronary artery disease. Arch Gen Psychiatry 2012;69:244255.

19 Teicher MH, Glod C, Cole JO: Emergence of intense suicidal preoccupation during fluoxetine treatment. Am J Psychiatry 1990;147: 207-210.

20 Gibbons RD, Brown H, Hur K, Davis JM, Mann JJ: Suicidal thoughts and behavior with antidepressant treatment. Reanalysis of the randomized placebo-controlled studies of fluoxetine and venlafaxine. Arch Gen Psychiatry 2012;69:580-587.

21 Fava M, Mischoulon D, Iosifescu D, Witte J, Pencina M, Flynn M, Harper L, Levy M, Rickels K, Pollack M: A double-blind, placebocontrolled study of aripiprazole adjunctive to antidepressant therapy among depressed outpatients with inadequate response to prior antidepressant therapy (ADAPT-A Study). Psychother Psychosom 2012;81:87-97.

22 Tomba E: Nowhere patients. Psychother Psychosom 2012;81:69-72.

23 Zuidersma M, Ormel J, Conradi HJ, de Jonge $\mathrm{P}$ : An increase in depressive symptoms after myocardial infarction predicts new cardiac events irrespective of depressive symptoms before myocardial infarction. Psychol Med 2012;42:683-693

24 Myers V, Gerber Y, Benyamini Y, Goldbourt U, Drory Y: Post-myocardial infarction depression: increased hospital admissions and reduced adoption of secondary prevention measures - A longitudinal study. J Psychosom Res 2012:72; 5-10.

25 Thombs BD, Ziegelstein RC: When prevention is a bad idea: problems with the DECARD trial and the premise behind it. J Psychosom Res 2012;72:333-335.

26 Klein RG, Mannuzza S, Olazagasti MAR, Roizen E, Hutchinson JA, Lashua EC, Castellanos FX: Clinical and functional outcome of childhood attention-deficit/hyperactivity disorder 33 years later. Arch Gen Psychiatry 2012;69: 1295-1303. 